\title{
Análise da eficiência na utilização de recursos da saúde nos maiores municípios
}

\section{brasileiros}

\author{
Analysis of the efficiency in the use of health resources in the largest brazilian municipalities \\ Análisis de la eficiencia en el uso de los recursos de salud en los mayores municipios brasileños
}

Recebido: 01/11/2021 | Revisado: 09/11/2021 | Aceito: 17/11/2021 | Publicado: 28/11/2021

\author{
Alessandro Lepchak \\ ORCID: https://orcid.org/ 0000-0003-0533-0386 \\ Universidade Estadual do Centro-Oeste, Brasil \\ E-mail: lepchak@gmail.com \\ Saulo Silva Lima Filho \\ ORCID: https://orcid.org/0000-0003-2199-8386 \\ Universidade Federal do Paraná, Brasil \\ E-mail: sauloslfilho@gmail.com \\ Eduardo Oliveira da Silva \\ ORCID: https://orcid.org/ 0000-0002-7260-3068 \\ Universidade Federal do Paraná, Brasil \\ E-mail: eduardoadv77@gmail.com \\ Blênio Cezar Severo Peixe \\ ORCID: https://orcid.org/0000-0001-8271-0628 \\ Universidade Federal do Paraná, Brasil \\ E-mail: bleniocsp@gmail.com
}

\begin{abstract}
Resumo
Este estudo analisa em que medida, os recursos destinados à saúde pública nos 80 maiores municípios brasileiros são utilizados de modo eficiente. Trata-se de uma pesquisa que se baseia na busca pela eficiência governamental, advinda com o gerencialismo aplicado ao setor público. Nesse sentido, supõe-se que as instituições públicas precisam gerir seus recursos com economia, mantendo ou ampliando a qualidade dos serviços prestados. A investigação se concentra em 76 cidades com dados entre 2013 a 2017 e mensurou a eficiência por meio da Análise Envoltória de Dados (DEA). Foram considerados os gastos de saúde (inputs) e outras 6 variáveis de saída (outputs), sendo elas: autorização de internação hospitalar, óbitos, dias de permanência, valor médio das internações, valor dos serviços hospitalares e dias de internações. A modelagem DEA foi desenvolvida com a orientação a insumo pelo modelo BCC (retornos variáveis de escala). Os resultados do estudo demonstram que os municípios mais eficientes se concentram nas regiões sul e sudeste. Contudo, o principal achado demonstra que somente o aumento de despesas com saúde não corresponde a serviços mais eficientes. Os dados demonstram que a eficiência obtida está relacionada com a boa gestão dos recursos públicos e não necessariamente com o volume de recursos envolvidos. Acentue-se ainda que os gestores públicos podem utilizar os municípios elencados no resultado como benchmarking do modelo, de modo a potencializar os indicadores e aprimorar a gestão dos recursos públicos. Ademais, o estudo apresenta avanço da teoria em administração e contabilidade pública para a gestão social na área da saúde.
\end{abstract}

Palavras-chave: Eficiência na gestão pública; Recursos aplicados na saúde pública; Análise envoltória de dados.

\begin{abstract}
This study analyzes the extent to which medical resources for public health in the 80 largest Brazilian municipalities are used efficiently. This research is based on the search for government efficiency, arising from the managerialism developed in the public sector. In this sense, it is assumed that public institutions need their resources economically, maintaining or increasing the quality of services provided. The investigation focuses on 76 cities with data between 2013 to 2017 and measured efficiency through Data Envelopment Analysis (DEA). Health expenses (incomings) and 6 other outgoing variables (outflows) were considered, namely: Hospital Admission Authorization, deaths, days of stay, average value of admissions, value of hospital services and days of admissions. The DEA modeling was developed with input orientation by the BCC model (variable returns to scale). The results of the study demonstrate that the most efficient cities are concentrated in the south and southeast regions. However, the main demonstration of the finding is that only the increase in health expenses does not correspond to more efficient services. The data show that the efficiency obtained is related to the good management of public resources and not necessarily to the volume of administrative resources. It should also be emphasized that public managers can use the municipalities listed in the result as a benchmark for the model, in order to enhance the indicators and improve the management of public resources. Furthermore, the study is advancing the theory in public administration and accounting for social management in the health area.
\end{abstract}

Keywords: Efficiency in public management; Resources applied in public health; Data envelopment analysis. 


\begin{abstract}
Resumen
Este estudio analiza en qué medida se utilizan eficientemente los recursos médicos para la salud pública en los 80 municipios más grandes de Brasil. Esta investigación se basa en la búsqueda de la eficiencia gubernamental, derivada del gerencialismo desarrollado en el sector público. En este sentido, se asume que las instituciones públicas necesitan sus recursos económicamente, manteniendo o aumentando la calidad de los servicios prestados. La investigación se centra en 76 ciudades con datos entre 2013 y 2017 y la eficiencia medida a través del Análisis envolvente de datos (DEA). Se consideraron los gastos de salud (ingresos) y otras 6 variables de salida (egresos), a saber: Autorización de ingreso hospitalario, defunciones, días de estancia, valor medio de los ingresos, valor de los servicios hospitalarios y días de ingresos. El modelo DEA fue desarrollado con orientación de entrada por el modelo BCC (rendimientos variables a escala). Los resultados del estudio demuestran que las ciudades más eficientes se concentran en las regiones sur y sureste. Sin embargo, la principal demostración del hallazgo es que solo el aumento de los gastos en salud no se corresponde con servicios más eficientes. Los datos muestran que la eficiencia obtenida está relacionada con la buena gestión de los recursos públicos y no necesariamente con el volumen de recursos administrativos. También cabe destacar que los gestores públicos pueden utilizar los municipios enumerados en el resultado como referencia del modelo, con el fin de potenciar los indicadores y mejorar la gestión de los recursos públicos. Además, el estudio avanza la teoría en la administración pública y la contabilidad de la gestión social en el área de la salud.
\end{abstract}

Palabras clave: Eficiencia en la gestión pública; Recursos aplicados en salud pública; Análisis envolvente de datos.

\title{
1. Introdução
}

Os recursos aplicados na área da saúde no Brasil seguem um percentual mínimo. Os quais nem sempre refletem a eficiência na prestação dos serviços públicos, principalmente num país em que a economia e a sociedade se encontram em desenvolvimento, quando comparado com outras economias mundiais (Silva, Maciel, Chacon \& Oliveira, 2008).

Matias-Pereira (2018) destaca que a eficiência na administração pública representa uma relação entre os recursos aplicados e o produto final obtido. É a razão entre o esforço e o resultado, entre a despesa e os serviços disponibilizados à população. Tal destaque se aplica também à área da saúde pública, pois dados da Organização Pan-Americana de Saúde (OPAS/OMS, 2012) indicam que o Brasil apresenta muitas desigualdades socioeconômicas, o que representa um desafio para as políticas sociais.

Deste modo, parte dos recursos públicos visam cumprir certa eficiência em determinada área, neste escopo, exclusivamente a eficiência técnica dos gastos com saúde. A literatura contemporânea sobre eficiência mostra a existência de uma correlação positiva entre o gasto total em saúde e o estado (indicadores) de saúde da população. No entanto, observa-se que regiões com valores semelhantes de investimento em saúde apresentam resultados substancialmente diferentes, o que levanta a questão sobre os níveis de eficiência e justifica a presente investigação (Geri et al., 2017).

Para a OMS (2010) todos os países ou regiões, independentemente do seu nível de rendimento, podem tomar medidas para reduzir a ineficiência nos serviços de saúde, a partir da avaliação local das causas implicadas nesse processo. Esta avaliação necessita da confrontação dos valores aplicados nos serviços e programas de saúde com resultados medidos pelo governo na forma de indicadores. Para tanto, uma avaliação de eficiência se mostra adequada aos pressupostos apresentados sendo objeto de estudo por diversos autores, dentre eles Sousa e Ramos (1999); Rezende, Slomski e Corrar (2005); Varela, Martins e Favero (2010); Wilbert e D’Abreu (2013); Gemelli, Savian e Bezerra (2013); Kaveski, Mazzioni e Hein (2013); Purohit (2014); Mazon, Mascarenhas e Dallabrida (2015); e Geri et al. (2017).

Este entendimento sobre a adoção de procedimentos eficientes no âmbito público ganhou força a partir da Reforma Gerencial, que exige a adoção de medidas para melhorar a qualidade do serviço prestado à sociedade (Abrucio, 2006). No Brasil esse movimento é percebido no princípio da eficiência na administração pública resultante da Emenda Constitucional no ${ }^{\circ}$ 19/98.

A eficiência aplicada aos serviços públicos pode ser entendida como a relação ótima obtida quando se consegue realizar a melhor combinação dos custos, do tempo e da qualidade para obter os bens e serviços (Graciliano et al., 2010). Diante do exposto e considerando a importância dos entes municipais no atendimento de modo satisfatório à população, questiona-se: em que medida, os recursos destinados à saúde pública nos 80 maiores municípios brasileiros são utilizados de modo eficiente? 
Neste sentido, o estudo analisa em que medida, os recursos destinados à saúde pública nos 80 maiores municípios brasileiros são utilizados de modo eficiente.

Justifica-se o presente estudo pelo fato da análise de eficiência proporcionar escores de comparação e melhoria entre as variáveis de modo que os municípios investigados tenham benchmarking's para direcionar suas decisões. Importante ainda ressaltar a avaliação por meio de dados disponibilizados pelo SUS, permitindo averiguar se os repasses entre os entes federativos são suficientes para garantir um serviço adequado à população brasileira.

A análise dos maiores municípios também permite uma comparação entre regiões geográficas posicionadas no mapa brasileiro além de contemplar todas as capitais deste complexo sistema que é a saúde pública no Brasil. O estudo contribui ainda para profissionais, pesquisadores e governantes no sentido de corroborar na avaliação das políticas públicas vigentes voltadas à área da saúde pública brasileira.

\section{Revisão de Literatura}

\subsection{Saúde Pública no Brasil}

A função alocativa do estado é direcionada ao provimento dos bens e serviços à coletividade. Nesse exercício, pressupõe-se que o ente estatal atenda as demandas da sociedade de modo eficaz e eficiente (Macedo, Ferreira \& Cruz, 2011). Neste estudo em específico, a compreensão da função alocativa se faz necessária, pois contempla a eficiência dos gastos em saúde, área essencial para o bem-estar da população.

Com o processo de descentralização praticado pelo Governo Federal concedendo maior autonomia aos estados e municípios, emergiu a necessidade de otimizar os gastos municipais. $\mathrm{O}$ aumento da participação popular na gestão das políticas públicas trouxe ainda a exigência de maior qualidade nos serviços de saúde (Kaveski et al., 2013). Isso significa que, ao transferir recursos para estados e municípios, o estado transfere a capacidade de tomada de decisões para os gestores locais, sendo estes mais adequados para vislumbrar as necessidades específicas da sua região. Tal processo colabora ou deveria auxiliar na otimização dos investimentos em saúde pública.

Considerando ainda o processo de descentralização, destaque-se que os municípios aumentaram progressivamente suas contribuições com o orçamento da saúde, de outro lado, houve o decréscimo da participação da União (Araújo, Gonçalves \& Machado, 2017). No âmbito municipal percebe-se tal processo de forma mais acentuada, ou seja, os valores mais expressivos transitam nas contas municipais e cabe às prefeituras a responsabilização sobre a eficiência na alocação destes gastos. Um exemplo dessas medidas remonta aos investimentos públicos em saneamento que, segundo os estudos de Crispim, Rodrigues, Vieira, Pessoa e Fernandes (2020), estão inversamente relacionados aos indicadores de mortalidade infantil.

Portanto, diante dessa configuração institucional, tem-se um cenário estruturado que, mesmo com sua regulação e delegação das funções primárias da saúde do estado nacional, apresenta um desafio para os municípios. Os recursos destinados à saúde, apesar de possuírem limites obrigatórios para sua aplicação anual, muitas vezes não são suficientes para atender todas as demandas que a população anseia. Assim, faz-se necessária uma análise de eficiência para otimizar a utilização destes recursos.

\subsection{Administração Gerencial e Eficiência no Âmbito Público}

A administração pública passou a ser estudada formalmente por volta do século XX, num contexto em que teóricos e profissionais demonstraram atenção quanto à precariedade nas atividades exercidas por órgãos administrativos. Apesar da apreensão, os resultados da profissionalização da função pública só foram evidenciados ao final do século XIX, quando da exaltação do compromisso democrático norte-americano (Denhardt \& Catlaw, 2017). 
Essa evolução da atividade gerencial no estado permitiu que o orçamento passasse a considerar novos conceitos e práticas (Giacomoni, 2019). Deste modo, lançaram mão de melhores informações orçamentárias para tomada de decisão, possibilitando a alocação de recursos de forma programática e auferindo resultados consistentes aos anseios sociais (Ho, 2018).

Esse movimento, inserido no âmbito da Nova Administração Pública, trouxe paradigmas que rompem com os padrões tradicionalmente observados, em que o rigor burocrático é substituído pelo foco nos resultados (Mathiasen, 1999). Para tanto, são utilizados instrumentos para redução de custos, critérios de medição de produtividade e comparabilidade entre pares, na condução de procedimentos mais eficazes e econômicos (Ashraf \& Uddin, 2016).

Assumindo, portanto, a relevância dos aspectos orçamentários, enquanto vetores na condução de políticas públicas, cabe esclarecer que sua função transpõe o foro administrativo, formal, mas remete à concepção do planejamento, perfazendo o escopo de atuação governamental (Abreu \& Câmara, 2014; Santos, Jankoski, Oliveira \& Rasoto, 2017).

No que compete ao estudo em tela, os recursos orçamentários que custeiam as políticas de saúde precisam ser indicados em cumprimento às normas aplicáveis, além de se atentar ao que é politicamente viável (Fernández-Gutiérrez \& Walle, 2018). Assume-se assim a necessidade de uma gestão eficiente (Mathiasen,1999).

Logo, o uso institucional eficiente cria meios de fomentar os resultados positivos das políticas públicas (Denhardt \& Catlaw, 2017). Embora trate-se de um ambiente com práticas e objetivos análogos, Fernández-Gutiérrez e Walle (2018) trazem a individualidade do gestor como responsável nas políticas e atividades organizacionais, razão pela qual são vistas disparidades na qualidade dos outputs gerados entre instituições similares.

A partir desses apontamentos, acentua-se a eficiência de gestão como o gasto coerente de recursos em vista da satisfação social (Ho, 2018). Pressuposto que pode conduzir pesquisas acadêmicas pautadas sob o discurso da estabilização institucional decorrente da legitimidade de sua atuação, marcada por maior governança, acordos com parceiros estratégicos e a assunção de novos objetivos, decorrentes da cobrança advinda da sociedade (Wallmeler, Helmig \& Feeney, 2018)

\subsubsection{Eficiência dos Gastos Públicos}

Enquanto conceito, a eficiência gerencial é imparcial. Trata-se de um critério que torna homogênea a análise da atuação de diversas instituições (Denhardt \& Catlaw, 2017). De maneira mais pragmática, mediante a mensuração de eficiência, o conjunto de atividades desenvolvidas em uma instituição recebe uma valoração, permitindo sua avaliação e comparabilidade.

Saliente-se que além de ser um princípio previsto na própria carta magna, a eficiência assume uma obrigação dos gestores públicos para com a população, especialmente porque a sociedade é a fonte provedora de recursos públicos, tendo o direito de conhecer e usufruir desses recursos (Giacomoni, 2019). Todavia, a eficiência não pode ser entendida apenas como maximização do resultado, mas sim como um melhor exercício das missões de interesse coletivo. Essa perspectiva assume que o Estado tem o dever de obter a maior realização, com os menores ônus possíveis (Aragão, 2004). Portanto, a análise de eficiência é útil para os gestores por duas razões: primeiro, porque é capaz de indicar o potencial de melhoria de desempenho de cada setor e, segundo, por avaliar o desempenho de cada organização, definindo indicadores de eficiência. A partir disso, é possível estabelecer metas de desempenho diferenciadas (Machado Junior, Irffi \& Benegas, 2011).

Esse movimento de busca pela eficiência direciona a gestão pública como atividade necessariamente racional e instrumental, voltada a servir ao público, na justa proporção das necessidades coletivas, admite-se como inadmissível o comportamento administrativo negligente, contra produtivo e ineficiente (Modesto, 2014).

Arretche (2011) destaca que a importância da avaliação de eficiência para o planejamento e gestão das políticas reside na possibilidade de dar ao gasto público melhor alocação e uso mais racional. Deste modo, evidencia-se que, com o estreitamento dos recursos, é de fundamental importância ser eficiente. 
A avaliação entre os resultados obtidos e os custos envolvidos permite a melhoria no processo de ampliação da eficiência, especialmente pela racionalização de recursos públicos (Jesus, Dantas \& Silva, 2019). No mesmo sentido, mas de forma mais alinhada com o contexto da presente pesquisa, Barros, Silva e Prata (2021) apontam que, a partir da análise de desempenho e eficiência, ligados aos recursos em saúde pública, o ente governamental pode rever os insumos críticos necessários para melhoria da oferta de bens e serviços.

Portanto, a eficiência nos gastos públicos busca a melhor relação entre os dispêndios executados com os resultados obtidos. Isto é, não basta obter ótimos resultados se o custo das ações for igualmente penoso à sociedade. É preciso rever procedimentos e repensar os objetivos governamentais ao que é estritamente fundamental aos anseios públicos (Matias-Pereira, 2018; Giacomoni, 2019).

\section{Aspectos Metodológicos}

A análise de eficiência remete a uma pesquisa quantitativa, logo a pesquisa adota um posicionamento ontológico baseado na realidade observável, sendo a utilização dos gastos com saúde o objeto central da pesquisa. O estudo é classificado como descritivo quanto à classificação geral da pesquisa, bibliográfico pela adoção de fontes e referências baseadas em artigos, livros e demais obras consultadas e documental para construção da base de dados e análise a partir de documentos publicados pelo FINBRA e SUS.

A aplicação dos recursos destinados à saúde ocorre diretamente no âmbito municipal, tendo a origem dos recursos baseados nas receitas próprias dos municípios e também em virtude das transferências estaduais e federais. Assim, a população da presente pesquisa é composta dos 80 maiores municípios brasileiros. Tal escolha se deve pelo fato destes municípios contemplarem todas as regiões do país e por representarem parcela significativa da população brasileira (37,57\%). A linha de corte para a seleção dos 80 maiores foi populacional, pois saúde é serviço compulsório previsto na constituição federal, assim foi descartada uma seleção por renda per capita. Também se levou em conta o fato de selecionar todas as capitais, deste modo o recorte populacional realizado foi para municípios com mais de 348 mil habitantes.

A amostra deste trabalho foi composta por municípios que disponibilizaram nos portais DataSus e Finbra os dados necessários para a avaliação da eficiência em um período de 5 anos (2013 a 2017). Esta escolha se deu de modo que contempla períodos em que ocorreram eleições federais e municipais, sendo possível comparação dos escores de eficiência num horizonte temporal. A partir da fase de coleta, e com a exclusão de dados faltantes, a base de dados resultou em 76 municípios, segue distribuição regional no Figura 1, sendo que o estado de São Paulo apresenta um número maior de municípios (36 municípios $22,37 \%$ do total):

Figura 1: Percentuais de municípios analisados por região geográfica.

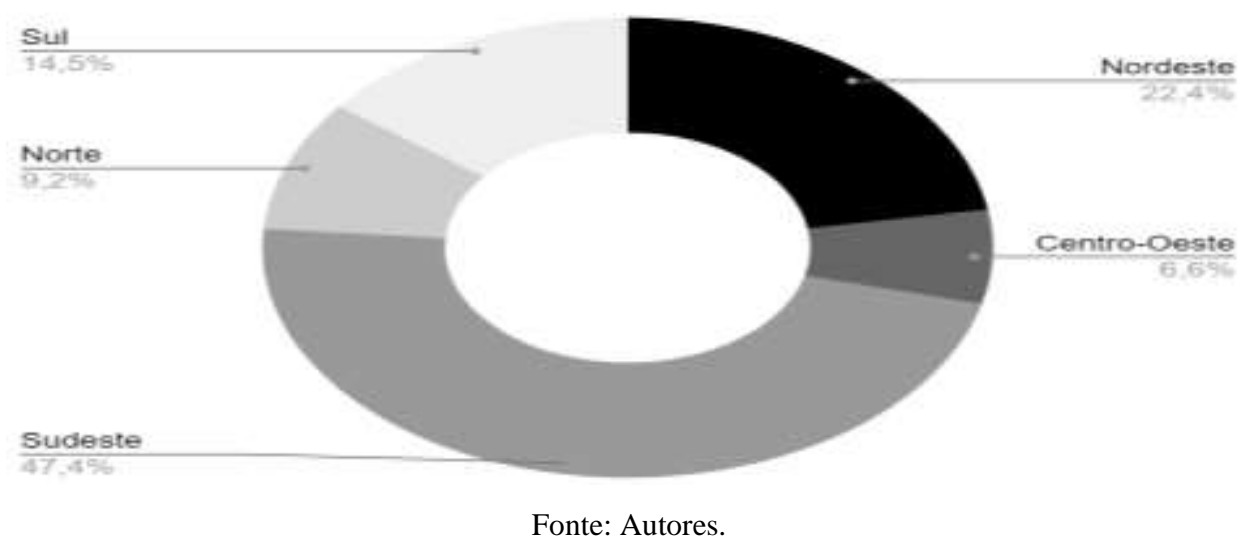


Estabelecida a amostra, cumpre destacar os procedimentos de análise que buscam atender ao objetivo de pesquisa. Nesse sentido, as etapas de análise foram dispostas de maneira que se compreendam as características dos dados, permita-se a mensuração da eficiência e sejam levantadas eventuais relações entre os gastos e os resultados de sua aplicação eficiente. Tais procedimentos são descritos no Quadro 1:

Quadro 1: Procedimentos de Pesquisa.

\begin{tabular}{|c|c|c|}
\hline Objetivo & Técnica Utilizada & Referência \\
\hline $\begin{array}{c}\text { Analisar de modo preliminar o comportamento dos das } \\
\text { variáveis }\end{array}$ & $\begin{array}{c}\text { Análise de tendência central, } \\
\text { verificação da dispersão dos dados e } \\
\text { análise interquartílica }\end{array}$ & Fávero e Belfiore (2017) \\
\hline Mensurar os escores de eficiência & Análise Envoltória de Dados (DEA) & $\begin{array}{c}\text { Charnes e Cooper (1984), } \\
\text { Bogetoft e Otto (2020) }\end{array}$ \\
\hline $\begin{array}{c}\text { Verificar a relação entre a eficiência na utilização dos } \\
\text { recursos e os resultados dos indicadores de saúde }\end{array}$ & Teste de Correlação de Pearson & Fávero e Belfiore (2017) \\
\hline
\end{tabular}

Nota: Os procedimentos relacionados à mensuração de eficiência tomaram como base a metodologia matemática desenvolvida por Charnes e Cooper (1984), enquanto a aplicação estatística dessa técnica utilizou o package desenvolvido por Bogetoft e Otto (2020). Fonte: Autores.

Como variáveis do estudo, faz-se necessária a separação entre variáveis de entrada (inputs) e de saída (outputs). Como variável de entrada foi utilizada a função 10 da classificação padrão da contabilidade pública no Brasil. A coleta ocorreu no site do Tesouro Nacional (Finbra) com os desdobramentos conforme Quadro 2:

Quadro 2: Funções e Sub-funções de Acordo com a Classificação Padrão da Contabilidade Pública.

\begin{tabular}{|c|c|}
\hline Função & Sub-função \\
\hline 10 & Saúde \\
\hline 10.301 & Atenção Básica \\
\hline 10.302 & Assistência Hospitalar e Ambulatorial \\
\hline 10.303 & Suporte Profilático e Terapêutico \\
\hline 10.304 & Vigilância Sanitária \\
\hline 10.305 & Vigilância Epidemiológica \\
\hline 10.306 & Alimentação e Nutrição \\
\hline
\end{tabular}

Fonte: FINBRA - Tesouro Nacional (2018).

A lógica da análise da eficiência utilizada seria minimizar inputs para maximizar outputs. Tal interpretação não faz inferências em pressupor reduções dos investimentos estatais em saúde, pelo contrário, suscita a discussão da eficiente aplicação dos recursos de modo a utilizar e otimizar todo valor investido pelos governos. Mazon et al. (2015) comentam que não é usual a redução do valor absoluto dos recursos colocados no setor saúde dos municípios. Pelo contrário, busca-se, frequentemente, a expansão desses recursos. Uma característica interessante do modelo é a possibilidade de se vislumbrar níveis ótimos de produção. Esses níveis ótimos podem servir como referência para a projeção de unidades em uma fronteira de eficiência. Como variáveis de saída (outputs) tem-se o Quadro 3: 
Quadro 3: Variáveis de Saída.

\begin{tabular}{|c|c|c|}
\hline Variável & $\begin{array}{c}\text { Sinal } \\
\text { Esperado }\end{array}$ & Descrição \\
\hline $\mathrm{AIH}(A I H)$ & + & $\begin{array}{l}\text { A Autorização de Internação Hospitalar é o instrumento de registro utilizado } \\
\text { por todos os gestores e prestadores de serviços SUS e apresenta como } \\
\text { característica a proposta de pagamento por valores fixos dos procedimentos } \\
\text { médicos. }\end{array}$ \\
\hline Óbitos (Obitos) & - & Número de óbitos ocorridos a partir das unidades de saúde \\
\hline $\begin{array}{l}\text { Dias de Permanência } \\
\quad \text { (Med_Permen) }\end{array}$ & + & $\begin{array}{l}\text { Unidade de medida que representa a assistência prestada a um paciente } \\
\text { internado durante um dia hospitalar. Avalia o tempo médio de } \\
\text { permanência (em dias) que um paciente permanece no hospital. }\end{array}$ \\
\hline $\begin{array}{l}\text { Valor Médio de } \\
\text { Internações } \\
\text { (Val_Med_int) }\end{array}$ & + & $\begin{array}{l}\text { Mede o gasto médio de recursos pagos pelo SUS na prestação de } \\
\text { atendimento hospitalar, na especialidade, a partir das Autorizações } \\
\text { para Internação Hospitalar (AIH). }\end{array}$ \\
\hline $\begin{array}{l}\text { Valor Serviços } \\
\text { Hospitalares } \\
(\text { Val_Serv_Hosp) }\end{array}$ & + & $\begin{array}{l}\text { Refere-se ao valor dos serviços hospitalares calculados a partir das AIH } \\
\text { aprovadas }\end{array}$ \\
\hline $\begin{array}{l}\text { Dias Internações } \\
\text { (Dias_Intern) }\end{array}$ & + & Total de dias de internações conforme às AIHs pagas no período \\
\hline
\end{tabular}

Fonte: ANVISA (2019); ANS (2019)

Considerou-se que as variáveis utilizadas possuem relação direta com o investimento realizado em saúde pública, de modo que apenas quando ligado aos Óbitos o coeficiente esperado é negativo. Isso se deve pela compressão que maiores gastos em saúde permitem reduzir o número de mortes (Marinho, 2003).

Para as demais variáveis, que supõem um coeficiente de relação positivo, propõe-se que o aumento de recursos governamentais na saúde possibilita maiores condições de atendimento à sociedade, seja na possibilidade de internação (AIH), no período de internação (Med_Perm), bem como na quantidade de dias pagos pelas internações (Dias_Intern) (Kaveski et al., 2013).

A análise de eficiência foi realizada por meio da metodologia de Análise Envoltória de Dados (DEA - Data Envelopment Analisys). Dentre trabalhos que utilizaram DEA para análise de eficiência na área da saúde destacam-se: Varela et al. (2010); Machado Junior et al. (2011); Gemelli, Savian e Bezerra (2013); Kaveski et al. (2013); e Mazon et al. (2015). Para este estudo utilizou-se o software R (versão v. 4.0.5), especialmente por meio da ferramenta desenvolvida por Bogetoft e Otto (2020).

Dito isso, saliente-se que a DEA foi criada por Farrel (1957) e aperfeiçoada por Banker (1993) e Charnes \& Cooper (1984). Tem por base a mensuração da eficiência e desempenhos relativos aferidos por meio da comparação de seus resultados (medidos em termos das quantidades geradas de seus diferentes produtos) e dos seus consumos (medidos pelos recursos que absorvem) com os resultados e os consumos das outras DMUs (neste estudo específico - os municípios) da amostra. Cabe frisar que os modelos básicos estão relacionados à retornos constantes de escala (CCR) e a retornos variáveis de escala (BCC) (Charnes \& Cooper, 1984). A análise na orientação-insumo utilizando o modelo BCC, cuja modelagem busca:

$\operatorname{Maximizar} \sum_{r=1}^{m} u_{r} y_{r k}-u_{k}$

Sujeito a

$\sum_{i=1}^{n} v_{i} x_{i k}=1$

$\sum_{r=1}^{m} u_{r} y_{r j}-\sum_{i=1}^{n} v_{i} x_{i k}-u_{k} \leq 0$

$u_{r} v_{i} \leq 0$

Em que

$y_{r}=$ quantidade de produto $\mathrm{r} ; x_{1}=$ quantidade de insumo $\mathrm{i}$; 
$\mathrm{u}, \mathrm{v}=\operatorname{pesos}$

$r=1, \ldots m ; i=1, \ldots, n ; j=1, \ldots, N$

Mazon et. al. (2015) complementam que a análise de envoltória de dados atribui a cada DMU um valor, representativo de seu desempenho relativo, estes escores variam entre 0 e 1, ou entre 0 e 100\%, e as unidades eficientes recebem valor igual a 1 ou $100 \%$. A DEA permite ainda, destacar em cada unidade, quais os níveis de consumo e de produção que tornaram as unidades eficientes (benchmarking).

Os escores de eficiência gerados a partir da análise envoltória de dados modelo BCC, orientação Graph (DEA-BCCGraph), foram calculados considerando a bissecção entre os valores viáveis e inviáveis (Bogetoft \& Otto, 2020). Ainda que menos precisa que as orientações à input ou output, esta premissa é necessária para que se tenha parâmetros na busca da fronteira eficiente e não significa ou indica uma sugestão para uma real redução de investimentos em saúde pública nos municípios.

\section{Análise dos Resultados}

Para iniciar a análise dos dados, a amostra foi submetida à interpolação linear de escassos dados ausentes, com o auxílio da ferramenta desenvolvida por Moritz e Bartz-Beielstein (2017), o que garantiu uma base de dados homogênea. Dito isso, ainda de modo preliminar, os dados parametrizados que compõem os escores de eficiência são descritos mediante estatísticas descritivas.

\subsection{Estatísticas descritivas}

Considerando os resultados apresentados pela Tabela 1, a partir da padronização dos dados pelo Z-score, assume-se certa similaridade no comportamento das variáveis. Isto é, no caso das medidas relativas à dispersão e às distâncias interquartílicas, observa-se que grande parte dos dados estão entre o terceiro quartil e o valor máximo. Ademais, o valor do desvio padrão reforça a volatilidade com que os dados se apresentam.

Por outro lado, é possível notar que os valores entre a média e a mediana não são nitidamente distintos, o que não afasta a hipótese de que o centro da distribuição esteja próximo à média dos dados.

Tais medidas amplas e distintas, reforçam a característica heterogênea em que os municípios brasileiros são compostos. Trata-se de uma amostra que, apesar de contar com grandes regiões urbanas, ainda apresenta distinções notáveis no que se refere à aplicação de recursos públicos, sobretudo, neste caso, aqueles ligados à saúde (Macedo, Ferreira \& Cruz, 2011; Espírito Santo, Fernando \& Bezerra, 2012).

Tabela 1: Estatísticas Descritivas.

\begin{tabular}{l|c|c|c|c|c|c|c}
\hline \multicolumn{1}{c}{ Min } & \multicolumn{1}{c}{$\mathbf{1}^{\circ}$ Quart } & \multicolumn{1}{c}{ Média } & \multicolumn{1}{c}{ Mediana } & \multicolumn{1}{c}{ Desv.Pad } & \multicolumn{2}{c}{ 3 $^{\circ}$ Mart } \\
\hline Saude & $-0,5874$ & $-0,3935$ & 0,0000 & $-0,2360$ & 0,9947 & $-0,0104$ & 7,4307 \\
\hline AIH & $-0,6676$ & $-0,4412$ & 0,0000 & $-0,3255$ & 0,9947 & $-0,0344$ & 6,6748 \\
\hline Obitos & $-6,6810$ & 0,0173 & 0,0000 & 0,2835 & 0,9947 & 0,4248 & 0,6816 \\
\hline Med_Permen & $-2,3843$ & $-0,6306$ & 0,0000 & $-0,1854$ & 0,9947 & 0,4688 & 3,9148 \\
\hline Val_Med_int & $-2,3020$ & $-0,6832$ & 0,0000 & $-0,0265$ & 0,9947 & 0,7713 & 3,6508 \\
\hline Val_Serv_Hosp & $-0,6299$ & $-0,4549$ & 0,0000 & $-0,3182$ & 0,9947 & $-0,0031$ & 6,7624 \\
\hline Dias_Intern & $-0,6742$ & $-0,4556$ & 0,0000 & $-0,3311$ & 0,9947 & $-0,0015$ & 6,1671 \\
\hline
\end{tabular}

Nota: Valores padronizados de acordo com o Z-score de cada variável. Fonte: Autores.

Quando os recursos são agrupados de acordo com a quantidade de municípios analisados por estado, fica evidente como o estado de São Paulo concentra grande parte dos grandes municípios envolvidos. Em outras palavras, mais de $25 \%$ dos 
municípios, que foram objeto do estudo, estão presentes no estado. Todavia, isso não significa que os recursos foram destinados ao estado da mesma forma.

Observe-se que a média dos recursos disponíveis aos estados do Rio de Janeiro e Minas Gerais, são superiores ao próprio estado de São Paulo. Isso pode ser explicado pela diluição do valor investido pela capital paulista em municípios potencialmente menores que a maior cidade paulista. Ao contrário do que ocorre com os outros estados que, proporcionalmente, não são tão distintos.

Tabela 2: Valores Médios das Variáveis por Estado/Unidade da Federação.

\begin{tabular}{|c|c|c|c|c|c|c|c|c|}
\hline Estado & $\mathbf{N}^{\circ}$ Munic* $^{*}$ & Saude $* *$ & $A I H^{*}$ & Obitos & Med_Permen & Val_Med_int & Val_Serv_Hosp $* *$ & Dias_Intern $* *$ \\
\hline SP & 20 & $9.686,81$ & 0,07 & $-3.202,87$ & 6,15 & $1.314,78$ & 81,39 & 0,41 \\
\hline RJ & 7 & $51.075,50$ & 0,06 & $-3.704,86$ & 7,95 & $1.040,09$ & 56,15 & 0,51 \\
\hline MG & 6 & $11.889,91$ & 0,07 & $-2.851,03$ & 6,51 & $1.716,35$ & 105,45 & 0,46 \\
\hline PE & 5 & 287,58 & 0,07 & $-3.321,44$ & 5,77 & $1.196,51$ & 90,61 & 0,44 \\
\hline PR & 4 & 599,92 & 0,07 & $-2.619,80$ & 5,79 & $1.640,95$ & 91,83 & 0,36 \\
\hline RS & 4 & 592,93 & 0,07 & $-3.042,40$ & 7,83 & $1.743,74$ & 102,04 & 0,51 \\
\hline GO & 3 & 538,97 & 0,07 & $-2.379,40$ & 6,65 & $1.676,04$ & 82,52 & 0,37 \\
\hline BA & 3 & 498,29 & 0,09 & $-3.915,80$ & 6,43 & $1.371,77$ & 117,47 & 0,65 \\
\hline SC & 3 & 322,37 & 0,04 & $-1.636,20$ & 5,34 & $2.050,58$ & 54,59 & 0,20 \\
\hline ES & 3 & 166,69 & 0,03 & $-968,40$ & 6,80 & $1.534,77$ & 32,75 & 0,17 \\
\hline
\end{tabular}

Nota: * Corresponde à $76 \%$ da amostra analisada no estudo; ** Valores em milhões de reais. Fonte: Autores.

Dados os resultados apresentados anteriormente, os valores por região demonstram que a região sudeste é de fato a região onde se concentra o maior volume absoluto investido em saúde pública. Entretanto, não são notadas distinções evidentes entre todo esse investimento e os resultados obtidos pelas demais regiões. Nesse sentido, cabe destacar que, por exemplo, a região sul obteve melhores indicadores nos óbitos e nos valores dos serviços hospitalares.

Tabela 3: Valores Médios das Variáveis por Região.

\begin{tabular}{|c|c|c|c|c|c|c|c|c|}
\hline Região & $\mathbf{N}^{\circ}$ Munic* & Saude** & AIH** & Obitos** & Med_Permen & Val_Med_int*** & Val_Serv_Hosp** & Dias_Intern*** \\
\hline Sudeste & 36 & $17.308,4505$ & 0,0624 & $-0,0031$ & 6,6150 & 0,0013 & 76,4396 & 0,4183 \\
\hline Norte & 7 & $14.481,4378$ & 0,0544 & $-0,0020$ & 5,8029 & 0,0010 & 45,2730 & 0,3194 \\
\hline Nordeste & 17 & $4.025,2824$ & 0,0826 & $-0,0037$ & 6,4706 & 0,0013 & 97,3547 & 0,5652 \\
\hline Centro-Oeste & 5 & 620,5020 & 0,0627 & $-0,0026$ & 6,5080 & 0,0017 & 81,5891 & 0,3679 \\
\hline Sul & 11 & 521,6807 & 0,0580 & $-0,0025$ & 6,4055 & 0,0018 & 85,3825 & 0,3706 \\
\hline
\end{tabular}

Nota: * Corresponde à $100 \%$ da amostra analisada no estudo; ** Valores em milhões de reais. Fonte: Autores.

Entretanto, para evidenciar de modo mais assertivo a relação entre o investimento em saúde e os resultados obtidos é preciso lançar mão da metodologia DEA, que traz o conceito de eficiência à discussão proposta. Saliente-se que introduzir a eficiência dentre os parâmetros de análise da qualidade na prestação de serviços públicos é inerente às propostas advindas com ao gerencialismo no setor público (Mathiasen, 1999; Denhardt \& Catlaw, 2017). Em outras palavras, não basta investir altas cifras de recursos públicos em sub-funções ligadas à saúde pública, mas é preciso que os resultados desses investimentos sejam 
observáveis. Nesse ponto, o gerencialismo traz um novo componente à gestão, exigindo que além de criar mais benefícios à sociedade, eles, eventualmente, custem menos (Ho, 2018; Matias-Pereira, 2018).

\subsection{Mensuração dos Escores de Eficiência}

Após os esclarecimentos necessários sobre a composição dos dados mediante análises descritivas, procedeu-se com a mensuração dos escores de eficiência. Acentue-se que foi utilizada a metodologia BCC, cujos resultados estão dispostos na Tabela 4.

Tabela 4: Escores de Eficiência dos Maiores Municípios Brasileiros.

\begin{tabular}{|c|c|c|c|c|c|c|c|}
\hline Município & UF & Região & 2013 & 2014 & 2015 & 2016 & 2017 \\
\hline Ananindeua & PA & Norte & 1,0000 & 0,8522 & 0,7257 & 0,7047 & 0,9789 \\
\hline Anápolis & GO & Centro-Oeste & 0,5979 & 0,5264 & 0,5750 & 0,5324 & 0,5473 \\
\hline Aparecida de Goiânia & GO & Centro-Oeste & 0,9127 & 0,9448 & 0,9373 & 1,0000 & 0,9042 \\
\hline Aracaju & $\mathrm{SE}$ & Nordeste & 0,3033 & 0,5965 & 0,1000 & 0,0815 & 0,0995 \\
\hline Bauru & SP & Sudeste & 0,6792 & 0,4724 & 0,4584 & 0,5223 & 0,5055 \\
\hline Belém & PA & Norte & 0,2317 & 0,2021 & 0,2452 & 0,2517 & 0,2854 \\
\hline Belford Roxo & RJ & Sudeste & 0,9108 & 0,9267 & 1,0000 & 0,9328 & 1,0000 \\
\hline Belo Horizonte & MG & Sudeste & 0,9515 & 1,0000 & 1,0000 & 0,9815 & 1,0000 \\
\hline Betim & MG & Sudeste & 0,9249 & 0,9112 & 0,9036 & 0,8010 & 0,7537 \\
\hline Blumenau & $\mathrm{SC}$ & Sul & 1,0000 & 1,0000 & 0,9565 & 1,0000 & 1,0000 \\
\hline Boa Vista & $\mathrm{RR}$ & Norte & 1,0000 & 0,9538 & 0,9172 & 0,8102 & 0,7954 \\
\hline Campina Grande & $\mathrm{PB}$ & Nordeste & 0,0405 & 0,1000 & 0,2291 & 0,0988 & 0,1000 \\
\hline Campinas & $\mathrm{SP}$ & Sudeste & 0,3743 & 0,4313 & 0,4431 & 0,4076 & 0,4348 \\
\hline Campo Grande & MS & Centro-Oeste & 0,2292 & 0,3467 & 0,4441 & 0,5130 & 0,5897 \\
\hline Campos dos Goytacazes & $\mathrm{RJ}$ & Sudeste & 0,8504 & 0,9712 & 0,9858 & 0,9635 & 0,8982 \\
\hline Canoas & $\mathrm{RS}$ & Sul & 0,5450 & 0,4410 & 0,4667 & 0,4922 & 0,4366 \\
\hline Carapicuíba & SP & Sudeste & 0,8503 & 0,8245 & 0,8230 & 0,8027 & 0,8050 \\
\hline Cariacica & ES & Sudeste & 1,0000 & 1,0000 & 1,0000 & 1,0000 & 1,0000 \\
\hline Caruaru & $\mathrm{PE}$ & Nordeste & 0,6650 & 0,6241 & 0,4029 & 0,4143 & 0,3797 \\
\hline Caucaia & $\mathrm{CE}$ & Nordeste & 0,9006 & 0,9054 & 0,9116 & 0,9222 & 0,9046 \\
\hline Caxias do Sul & $\mathrm{RS}$ & Sul & 0,8606 & 0,9241 & 0,7534 & 0,7368 & 0,7159 \\
\hline Contagem & MG & Sudeste & 0,7882 & 0,7886 & 0,7742 & 0,7853 & 0,7412 \\
\hline Cuiabá & MT & Centro-Oeste & 0,1000 & 0,1000 & 0,1000 & 0,1000 & 0,2369 \\
\hline Curitiba & PR & Sul & 1,0000 & 0,9279 & 1,0000 & 0,9896 & 1,0000 \\
\hline Diadema & SP & Sudeste & 0,6805 & 0,7043 & 0,6794 & 0,6601 & 0,6536 \\
\hline Duque de Caxias & RJ & Sudeste & 0,4526 & 0,0842 & 0,1000 & 0,0043 & 0,1000 \\
\hline Feira de Santana & $\mathrm{BA}$ & Nordeste & 0,3721 & 0,4528 & 0,3501 & 0,2944 & 0,3763 \\
\hline Florianópolis & $\mathrm{SC}$ & Sul & 0,4509 & 0,3178 & 0,3245 & 0,3190 & 0,3534 \\
\hline Fortaleza & $\mathrm{CE}$ & Nordeste & 0,8643 & 0,9173 & 0,9598 & 1,0000 & 0,9874 \\
\hline Franca & SP & Sudeste & 0,7011 & 0,6505 & 0,7026 & 0,5910 & 0,6054 \\
\hline Goiânia & GO & Centro-Oeste & 0,8749 & 0,8107 & 0,7922 & 0,7468 & 0,7929 \\
\hline Guarulhos & SP & Sudeste & 0,6178 & 0,6221 & 0,5576 & 0,4445 & 0,3769 \\
\hline Itaquaquecetuba & SP & Sudeste & 0,7673 & 0,7414 & 0,7713 & 0,6935 & 0,7249 \\
\hline Jaboatão dos Guararapes & $\mathrm{PE}$ & Nordeste & 0,7529 & 0,7045 & 0,6866 & 0,6899 & 0,7522 \\
\hline João Pessoa & $\mathrm{PB}$ & Nordeste & 0,2576 & 0,1000 & 0,1000 & 0,1000 & 0,2541 \\
\hline Joinville & $\mathrm{SC}$ & Sul & 0,4293 & 0,4837 & 0,5186 & 0,4381 & 0,4569 \\
\hline Juiz de Fora & MG & Sudeste & 1,0000 & 0,8939 & 0,6988 & 0,7033 & 0,7072 \\
\hline Jundiaí & SP & Sudeste & 0,5461 & 0,5258 & 0,5236 & 0,4986 & 0,5255 \\
\hline Londrina & $\mathrm{PR}$ & Sul & 0,3033 & 0,4447 & 0,5071 & 0,4632 & 0,4924 \\
\hline Macapá & AP & Norte & 0,7513 & 0,7273 & 0,7384 & 0,7559 & 0,7933 \\
\hline Maceió & $\mathrm{AL}$ & Nordeste & 0,4947 & 0,3841 & 0,1000 & 0,1000 & 0,1000 \\
\hline Manaus & $\mathrm{AM}$ & Norte & 1,0000 & 1,0000 & 0,8174 & 0,4236 & 0,4858 \\
\hline Maringá & $\mathrm{PR}$ & Sul & 0,4776 & 0,4853 & 0,4837 & 0,4980 & 0,4753 \\
\hline Mauá & SP & Sudeste & 0,7011 & 0,6880 & 0,6832 & 0,6520 & 0,7266 \\
\hline Mogi das Cruzes & SP & Sudeste & 0,4662 & 0,4608 & 0,3463 & 0,2732 & 0,2261 \\
\hline Montes Claros & MG & Sudeste & 0,3702 & 0,3792 & 0,3199 & 0,2738 & 0,3986 \\
\hline
\end{tabular}




\begin{tabular}{|c|c|c|c|c|c|c|c|}
\hline Natal & $\mathrm{RN}$ & Nordeste & 0,7969 & 1,0000 & 0,8731 & 0,6404 & 0,5517 \\
\hline Niterói & RJ & Sudeste & 0,9877 & 1,0000 & 1,0000 & 1,0000 & 0,8222 \\
\hline Olinda & $\mathrm{PE}$ & Nordeste & 1,0000 & 0,9022 & 0,8886 & 0,8888 & 0,8962 \\
\hline Osasco & SP & Sudeste & 0,5426 & 0,5244 & 0,5076 & 0,5168 & 0,5237 \\
\hline Pelotas & $\mathrm{RS}$ & Sul & 0,7969 & 0,7757 & 0,7360 & 0,7875 & 0,9176 \\
\hline Petrolina & $\mathrm{PE}$ & Nordeste & 0,6422 & 0,6716 & 0,6812 & 0,6696 & 0,8236 \\
\hline Piracicaba & $\mathrm{SP}$ & Sudeste & 0,5907 & 0,5557 & 0,5811 & 0,6127 & 0,6461 \\
\hline Ponta Grossa & PR & Sul & 0,6205 & 0,5979 & 0,5688 & 0,5356 & 0,5433 \\
\hline Porto Alegre & RS & Sul & 0,9799 & 0,9978 & 1,0000 & 0,9489 & 0,9637 \\
\hline Porto Velho & RO & Norte & 0,7278 & 0,4409 & 0,5073 & 0,5678 & 0,6052 \\
\hline Recife & $\mathrm{PE}$ & Nordeste & 1,0000 & 1,0000 & 0,9922 & 1,0000 & 1,0000 \\
\hline Ribeirão Preto & SP & Sudeste & 0,9320 & 0,8978 & 0,8677 & 0,8310 & 0,7583 \\
\hline Rio Branco & $\mathrm{AC}$ & Norte & 0,6399 & 0,6691 & 0,5723 & 0,5916 & 0,6467 \\
\hline Rio de Janeiro & RJ & Sudeste & 1,0000 & 1,0000 & 0,9366 & 0,7995 & 0,8116 \\
\hline Salvador & $\mathrm{BA}$ & Nordeste & 0,9630 & 1,0000 & 0,9320 & 1,0000 & 1,0000 \\
\hline Santo André & SP & Sudeste & 0,2615 & 0,2182 & 0,2089 & 0,2089 & 0,2812 \\
\hline Santos & SP & Sudeste & 0,4599 & 0,4641 & 0,4230 & 0,4344 & 0,4376 \\
\hline São Bernardo do Campo & SP & Sudeste & 0,6097 & 0,5876 & 0,5738 & 0,5242 & 0,5160 \\
\hline São Gonçalo & RJ & Sudeste & 0,9116 & 0,4863 & 0,4227 & 0,3165 & 0,4529 \\
\hline São João de Meriti & RJ & Sudeste & 0,8545 & 0,8729 & 0,8576 & 0,8305 & 0,8373 \\
\hline São José dos Campos & SP & Sudeste & 0,5598 & 0,5795 & 0,3528 & 0,3084 & 0,3594 \\
\hline São Luís & MA & Nordeste & 1,0000 & 0,9395 & 0,9367 & 0,9145 & 1,0000 \\
\hline São Paulo & SP & Sudeste & 1,0000 & 1,0000 & 0,9838 & 1,0000 & 1,0000 \\
\hline São Vicente & SP & Sudeste & 0,7892 & 0,7694 & 0,7683 & 0,7734 & 0,7619 \\
\hline Serra & ES & Sudeste & 0,8856 & 0,8696 & 0,6886 & 0,6403 & 0,5587 \\
\hline Sorocaba & $\mathrm{SP}$ & Sudeste & 1,0000 & 0,8486 & 0,7020 & 0,6899 & 0,3987 \\
\hline Teresina & PI & Nordeste & 0,1000 & 0,1000 & 0,1000 & 0,1000 & 0,1000 \\
\hline Uberlândia & MG & Sudeste & 1,0000 & 0,5038 & 0,4960 & 0,5312 & 0,5988 \\
\hline Vitória & ES & Sudeste & 0,7634 & 1,0000 & 0,5844 & 0,7098 & 0,9158 \\
\hline Vitória da Conquista & $\mathrm{BA}$ & Nordeste & 0,5670 & 0,5484 & 0,5011 & 0,4707 & 0,5199 \\
\hline \multicolumn{3}{|l|}{ Mínimo } & 0,0405 & 0,0842 & 0,1000 & 0,0043 & 0,0995 \\
\hline \multicolumn{3}{|l|}{ Média } & 0,6978 & 0,6733 & 0,6337 & 0,6093 & 0,6280 \\
\hline \multicolumn{3}{|l|}{ Desvio Padrão } & 0,2646 & 0,2704 & 0,2710 & 0,2762 & 0,2659 \\
\hline \multicolumn{3}{|l|}{ Coeficiente de Variação } & 0,3793 & 0,4016 & 0,4276 & 0,4532 & 0,4235 \\
\hline \multicolumn{3}{|l|}{ Máximo } & 1,0000 & 1,0000 & 1,0000 & 1,0000 & 1,0000 \\
\hline
\end{tabular}

Nota: Escores gerados a partir da variável de entrada Gastos com a função Saúde e variáveis de saída Autorizações para internações, Óbitos, Média de permanência em dias, Valor Médio de Internações, Valor dos Serviços Hospitalares e Dias de Internações. Fonte: Autores.

A partir dos resultados, observa-se que os municípios de Cariacica, Recife, São Paulo, Blumenau, Belo Horizonte, Curitiba, Salvador, Porto Alegre, Niterói e São Luís apresentaram os dez melhores escores agregados de eficiência no período analisado, resultados de benchmarking's convergentes com o estudo efetuado por Souza e Barros (2013). Isto é, encontraram a relação ideal entre o volume de despesas utilizados em saúde pública e os resultados nos indicadores avaliados.

Dos municípios que atingiram essa marca de eficiência, 70\% estão concentrados entre as regiões sul e sudeste, ao passo que os outros $30 \%$ encontram-se na região nordeste. Ademais, é imperativo destacar que o uso da metodologia DEA não supõem que altos investimentos em saúde (input) remontam em um melhor escore de eficiência. Como é o caso dos municípios de Cariacica (ES) e Niteroi (RJ) que apresentaram investimentos relativamente baixos, mas que atenderam de modo eficiente sua população.

Por outro lado, os municípios de Montes Claros, Belém, Maceió, Aracaju, Santo André, João Pessoa, Teresina, Duque de Caxias, Cuiabá e Campina Grande, obtiveram os menores escores. Estes municípios apresentaram investimentos moderados em saúde, mas os resultados de suas ações foram insuficientes frente aos resultados obtidos pelos seus pares.

Cabe citar ainda que, sessenta por cento desses municípios estão presentes na região norte e nordeste, outros $30 \%$ na região sudeste e apenas um município é da região centro-oeste. Todavia, a análise sobre os piores e melhores municípios não é 
capaz de refletir os resultados em escopo mais amplo, como na análise entre regiões ao longo dos períodos. Sob essa perspectiva de análise comparativa entre regiões, o Figura 2 demonstra como os escores de eficiência se comportaram ao longo dos exercícios avaliados. É possível verificar a regularidade nos resultados obtidos na região sul, enquanto as regiões nordeste e centro-oeste nitidamente alternam seus resultados. Em outras palavras, no momento que a região centro-oeste melhorou seus escores a região nordeste apresentou ligeira queda em seus indicadores.

Figura 2: Média dos Escores de Eficiência entre as Regiões.

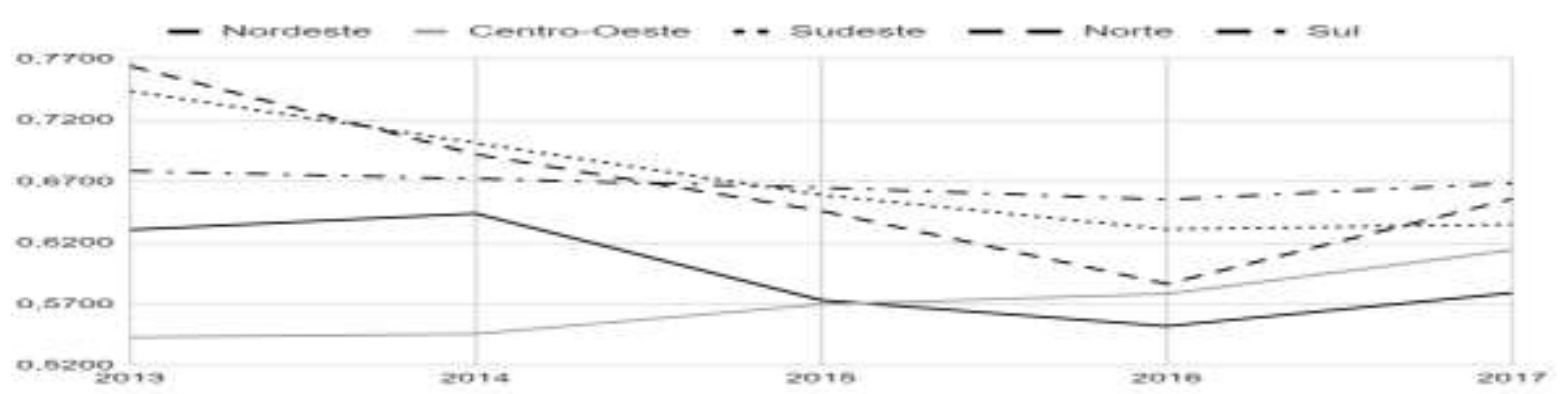

Fonte: Autores.

Os resultados da região centro-oeste se aproximam da região sudeste que também demonstrou queda em seus escores de eficiência ao longo dos anos. Cumpre ainda destacar os resultados verificados na região norte que, após uma brusca queda de eficiência até 2016, conseguiu melhorar seus resultados no ano seguinte. Ao considerar todas as regiões, os resultados demonstram queda na eficiência em saúde pública ao longo dos anos, em torno de 10\% na comparação entre 2013 para 2017. Este resultado é notado por meio do Figura 3, no qual nota-se que a média dos escores de eficiência municipal se mantiveram entre 0,60 e 0,69 .

A queda nos resultados se deve em grande parte pela redução nos escores máximos ao longo dos anos. Enquanto 14 municípios obtiveram o escore máximo em 2013, apenas 9 obtiveram o mesmo resultado em 2017, uma redução de 35,71\%.

Figura 3: Média dos Escores de Eficiência ao Longo do Período Analisado.

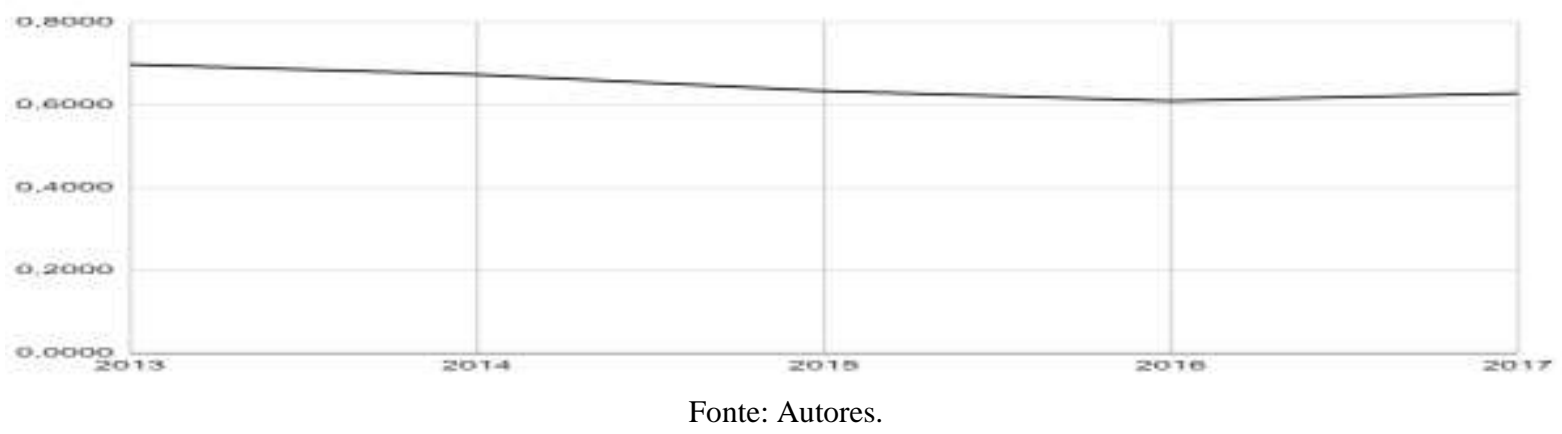

Os municípios considerados modelos para esta análise destacam-se por conseguirem melhores resultados nas variáveis de saída. Um exemplo desta constatação é a variável de saída "Obitos". Os municípios que mais vezes apareceram como benchmarking foram aqueles que conseguiram reduzir o número de falecimentos. Logo, o aumento nos investimentos em saúde, permite ampliar o número de dias de internamento, além de permitir que os municípios atendam a um maior número de pessoas e assim, melhor aplicar os recursos destinados à saúde.

A partir da análise envoltória de dados foi possível gerar a curva da fronteira eficiente. Pelo modelo BCC, a linha superior inclui municípios que estão na fronteira, sendo aqueles que logicamente atingiram $100 \%$ de eficiência. Abaixo da 
fronteira eficiente encontra-se a área de melhoria para as outras unidades de análise. Segue na Figura 4 demonstrando a fronteira eficiente para o ano de 2017:

Figura 4: Fronteira de Eficiência no Exercício de 2017

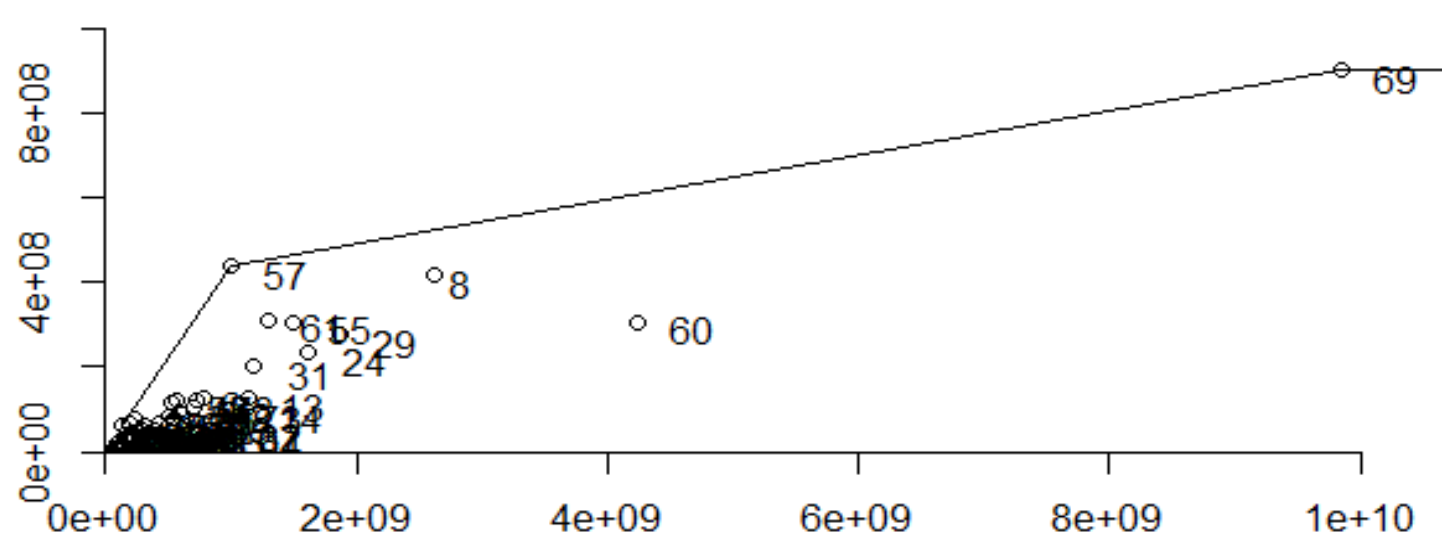

Nota: Visualmente não foram observadas diferenças significativas na composição das fronteiras de eficiência entre exercícios; Exemplos de escores na fronteira de eficiência: 8 = Belo Horizonte, 57 = Recife, 61 = Salvador, 69 = São Paulo. Fonte: Autores.

Por fim, a análise da eficiência perpassa algumas escolhas teóricas com relação às variáveis utilizadas, neste estudo foram utilizadas pesquisas anteriores a fim de facilitar tais escolhas. Nota-se que os municípios podem gerenciar melhor algumas destas variáveis, pois as mesmas terão impacto significativo na eficiência e alocação dos gastos com saúde. Em outras palavras, a fim de melhor gerir os gastos com saúde é essencial para os municípios o controle sobre a permanência dos pacientes nas unidades, bem como sobre os valores médios de internação e o número de AIH aprovadas. A relação entre estas variáveis acaba elevando a eficiência dos municípios e diretamente proporciona maior bem-estar à população.

Portanto, observa-se que os pressupostos defendidos pelo movimento gerencial trazem novas definições à atuação governamental, especialmente quanto ao uso consciente de recursos e o atendimento eficiente à sociedade (Denhardt \& Catlaw, 2017; Ho, 2018). Nessa seara, a variável de input, relacionada aos dispêndios na função saúde, representa o mecanismo de controle governamental programático que dispõe recursos de acordo com o planejamento e as demandas previamente estabelecidos (Giacomoni, 2019).

Na relação entre o orçamento aplicado, a fronteira de eficiência demonstra que não basta uma alocação econômica ou ainda melhores indicadores de resultados, mas sim uma estimação coerente, que disponha recursos de modo adequado e os coloque efetivamente em favor dos seus usuários, como defendido pela NAP (Dumitrescu \& Dogaru, 2016; Ashraf \& Uddin, 2016).

\subsection{Teste de correlação}

Em que pese seja possível identificar similaridades entre os escores de eficiência e os resultados ligados à saúde pública, o teste de correlação permite identificar se de fato a variação de determinado output, se comporta de modo significativamente similar à variação do escore de eficiência.

Nesse sentido, os resultados demonstram que, quanto mais recursos investidos em saúde melhores os indicadores, numa relação positiva de 0,2492 (p-valor =0,0000). Contudo, as relações com a "AIH" ( $\rho=0,3838$, p-valor =0,0000), "Med_Perman" $(\rho=0,4470$, p-valor $=0,0000)$, “Val_Med_int" $(\rho=0,5593$, p-valor $=0,0000)$, “Val_Serv_Hosp" $(\rho=0,4171$, p-valor $=0,0000)$ e "Dias_Intern" $(\rho=0,4161, \mathrm{p}$-valor $=0,000)$ demonstram que o investimento em saúde não é soberano para determinar os resultados à sociedade. 
Isso significa que a eficiência percebida pelos municípios é devida sobretudo à gestão dos indicadores envolvidos nos outputs. Já o aumento de recursos de modo isolado não traz consigo as melhorias esperadas. Reitere-se que este entendimento é consoante ao difundido pela Reforma Gerencial no âmbito público, em que a eficiência na administração governamental pressupõe uma melhoria nos serviços ofertados à sociedade sem, necessariamente, envolver o aumento de gastos (Matias-Pereira, 2018).

Os coeficientes encontrados condizem com as expectativas iniciais do presente estudo, inclusive quanto ao comportamento no número de óbitos que é reduzido à medida que os escores de eficiência são ampliados $(\rho=-0,3411, p$-valor $=0,0000$ ). Logo, assume-se que aumentando os investimentos e as condições de atendimento à população, o número de óbitos tende a diminuir.

Tais resultados vão ao encontro dos achados levantados por Crispim (et al, 2020), sobretudo quanto à capacidade dos investimentos públicos interferirem nos indicadores de saúde pública. Da mesma forma, os resultados encontrados denotam avanços no campo científico ao apontar a correlação entre saúde e determinados indicadores de saúde sensíveis a diversos municípios brasileiros. Outrossim, saliente-se que esses achados possuem respaldo inclusive em pesquisas anteriores como em Jesus, Dantas e Silva (2019) e Barros, Silva e Prata (2021) ao indicarem que a racionalização dos gastos público são relevantes na busca por melhor desempenho e eficiência nas ações governamentais.

Entretanto, diante desses resultados, cabe frisar que a gestão de recursos voltada à saúde pública não é simples e esbarra em especificidades regionais. Outrossim, a partir do estudo em tela, percebe-se que a gestão voltada para melhoria dos indicadores utilizados como outputs traz consigo uma melhoria inerente da qualidade do serviço prestado.

Como mencionado, somente o aporte de recursos financeiros não é suficiente para prover melhoria de eficiência (Arretche, 2011; Ho, 2018). É preciso que sua utilização seja percebida pelos usuários finalísticos, com disponibilidade para internação, recursos para as diárias necessárias, valores de tratamentos condizentes com as demandas, permitindo um acompanhamento tempestivo que reduza a taxa de mortalidade, como verificado nos municípios mais eficientes identifica neste estudo.

\section{Conclusões}

A busca pela prestação de serviços mais eficientes à sociedade é inerente ao movimento gerencial. Em outras palavras, o sucesso da gestão pública não está no volume de recursos investidos, mas se ocupa em avaliar a relação desses recursos com os resultados obtidos.

Sob esta perspectiva, o presente estudo mensurou a eficiência na utilização de recursos destinados à saúde pública nos 80 maiores municípios brasileiros. Por meio da metodologia de Análise Envoltória de Dados foi possível obter escores de eficiência para municípios que atendem grande parte da população nacional.

Como levantado pela revisão de literatura, a aplicação de altas cifras de recursos financeiros não corresponde, necessariamente, a um aumento na qualidade dos serviços prestados. De forma resumida, na pesquisa em tela, esses serviços envolvem a capacidade de autorizar a internação hospitalar, a quantidade de óbitos verificada, a possibilidade de manter o paciente internado pelo tempo necessário e os gastos envolvidos na internação hospitalar.

Os escores de eficiência são avaliados pela relação ideal entre o dispêndio de recursos na função orçamentária de saúde e os resultados observados. Neste sentido, os achados apontam que as regiões sul e sudeste concentram grande parte dos municípios mais eficientes. Entretanto, o mérito da pesquisa consiste em verificar, de modo empírico, que de fato a eficiência possui maior relação com os outputs gerados do que com os valores investidos.

Isto demonstra que a avaliação de eficiência precisa ser ampliada para constituir um critério imparcial de mensuração da qualidade nos serviços prestados, além de possibilitar a comparabilidade entre instituições, perspectivas defendidas pelo 
gerencialismo aplicado ao setor público. A partir desse levantamento, gestores podem basear suas decisões em benchmarking's, melhorando a qualidade nos serviços prestados à sociedade.

Por fim, reitera-se que o estudo em tela contribui ao trazer novos componentes à avaliação de organizações públicas que gerem recursos voltados à saúde. Além disso, reforça a tese de que a eficiência deve basear a condução das atividades públicas, como previsto desde a Reforma Gerencial.

Todavia, o estudo não é imune a limitações. A amostra utilizada não remete a um passado tão longínquo, mas poderá ser ampliada à medida que novos dados forem disponibilizados pelas instituições responsáveis. Além disso, podem ser utilizados critérios diretamente ligados aos repasses intergovernamentais, oferecendo uma maior robustez aos inputs inseridos à metodologia DEA.

\section{Referências}

Abreu, C. R., \& Câmara, L. M. (2014). O Orçamento Público Como Instrumento de Ação Governamental: uma análise de suas redefinições no contexto da formulação de políticas públicas de infraestrutura. Revista de Administração Pública. 49(1), 73-90.

Abrucio, F. L. (2006). Os Avanços e os Dilemas do Modelo Pós-Burocrático: A Reforma da Administração Pública à Luz da Experiência Internacional Recente. In Bresser Pereira, L.C., \& Spink, P.K. (Org). Reforma do Estado e Administração Pública Gerencial. (pp 173 - 199). FGV.

Aragão, A. S. de. (2004). O Princípio da Eficiência. Revista de Direito Administrativo, 24(3), 1-6.

Araújo, C. E. L., Gonçalves, G. Q., \& Machado, J. A. (2017). Os municípios brasileiros e os gastos próprios com saúde: algumas associações. Ciência \& Saúde Coletiva, 22(3), 953-963. https://doi.org/10.1590/1413-81232017223.15542016

Arretche, M. T. S. (2011). Tendências no estudo sobre avaliação. In Avaliação de Políticas Sociais: uma questão de debate. São Paulo: Cortez.

Ashraf, J. \& Uddin, S. (2016). New public management, costa saving and regressive effects: A casa from less developed country. Critical Perspectives on Accounting. 41(1), 18-33.

Banker, R. D. (1993). Maximum Likelihood, Consistency and Data Envelopment Analysis: A Statistical Foundation. Management Science, 39(10), 1265-1273. https://doi.org/10.1287/mnsc.39.10.1265.

Barros, L.O.; Silva, F. G. F.; \& Prata, B. T. (2021). Avaliação de desempenho e capacidade do sistema de saúde espanhol na pandemia de SARS-CoV-2. Research, Society and Development, 10(1), e53410111886. http://dx.doi.org/10.33448/rsd-v10i1.11886.

Bogetoft, P., \& Otto, L. (2020), Benchmarking with DEA and SFA, R package version 0.29.

Charnes, A., \& Cooper, W. W. (1984). Preface to topics in data envelopment analysis. Annals of Operations Research, 2(1), 59-94. https://doi.org/10.1007/BF01874733.

Crispim, D. L.; Rodrigues, R. S. S.; Vieira, A. S. de A.; Pessoa, F. C. L.; \& Fernandes, L. L. (2020). Health indicators in Amazonian cities: case study in the State of Pará, Brazil. Research, Society and Development, 9(7), e635973531. 10.33448/rsd-v9i7.3531.

Denhardt, R. B., \& Catlaw, T. J. (2017). Teorias da Administração Pública. (2a ed). Cengage Learning.

Dumitrescu, P. A., \& Dogaru, T. C. (2016). The challenges of the administrative process in a financial crisis and their effectiveness. Procedia Economics and Finance. 39 (1), 827-832.

Espírito Santo, A. C. G. do., Fernando, V. C. N., \& Bezerra, A. F. B. (2012). Despesa pública municipal com saúde em Pernambuco, Brasil, de 2000 a 2007. Ciência \& Saúde Coletiva, 17(4), 861-871. https://doi.org/10.1590/S1413-81232012000400009

Farrel, M. J. (1957). The Measurement of Productive Efficiency. Journal of the Royal Statistical Society, 120(3), 253-290.

Fávero, L.P. \& Belfiore, P. (2017). Manual de Análise de Dados. Elsevier.

Fernádez-Gutiérrez, M., \& Walle, S.V. (2018). Equity or Efficiency? Explaining Public Official's Values. Public Administration Review. 79(1), 25-34.

Gemelli Savian, M. P., \& Bezerra, F. M. (2013). Análise de eficiência dos gastos públicos com educação no ensino fundamental no Estado do Paraná. Economia \& Região, 1(1), 26-47. https://doi.org/10.5433/2317-627X.2013v1n1p26

Geri, M., Monterubbianesi, P. D., Lago, F. P., \& Moscoso, N. S. (2017). Eficiencia del Gasto Total en Salud: Análisis no paramétrico en una muestra amplia de países Total health expenditure efficiency: non-parametric analysis in a large sample of countries. Revista Salud Pública, 19(1), 79-85. https://doi.org/10.15446/rsap.v19n1.44546

Giacomoni, J. (2019). Orçamento Governamental: teoria, sistema, processo. Atlas.

Graciliano, E. A., Filho, J. C. M., Nunes, A. de P., Pontes, F. C. de M., \& Zampa, F. F. (2010). Accountability na administração pública federal: contribuição das auditorias operacionais do TCU. Pensar Contábil, 12(47), 43-51.

Ho, A. T. (2018). From Performance Budgeting to Performance Budget Management: Theory and Practice. Public Administration Review.1-11. 
Jesus, A. C. S.; Dantas, A. L. F.; \& Silva, M. V. (2019). A eficiência do gasto público em educação: Uma avaliação na região do Seridó Potiguar (2008-2015). Research, Society and Development, 8(12), e138121602. https://doi.org/10.33448/rsd-v8i12.1602.

Kaveski, I. D. S., Mazzioni, S., \& Hein, N. (2013). A eficiência na utilização de recursos no setor de saúde: uma análise dos municípios do oeste catarinense. Revista de Gestão Em Sistemas de Saúde, 2, 53-84. https://doi.org/10.5585/rgss.v2i2.72

Macedo, M. A. da S., Ferreira, A. C. de S., \& Cruz, C. F. da. (2011). Índice De Desenvolvimento Sustentável: Uma Análise Apoiada Em Dea Para Os Municípios do Estado do Rio de Janeiro. Gestão \& Regionalidade, 27(81), 19-31. https://doi.org/10.13037/gr.vol27n81.1108.

Machado Junior, S. P., Irffi, G., \& Benegas, M. (2011). Análise da Eficiência Técnica dos Gastos com Educação, Saúde e Assistência Social dos Municípios Cearenses. Planejamento e Políticas Públicas, (36), 87-113. http://www.repositorio.ufc.br/handle/riufc/814.

Marinho, A. (2003). Avaliação da eficiência técnica nos serviços de saúde nos municípios do Estado do Rio de Janeiro. Revista Brasileira de Economia, 57(3), 515-534. https://doi.org/10.1590/S0034-71402003000300002

Mathiasen, D.G. (1999). The New Public Management and Its Critics. International Public Management Journal. 2(1), 90-111.

Matias-Pereira, J. (2018). Administração Pública (5 ed). São Paulo: Atlas.

Mazon, L. M., Mascarenhas, L. P. G., \& Dallabrida, V. R. (2015). Eficiência dos gastos públicos em saúde: Desafio para municípios de Santa Catarina, Brasil. Saúde e Sociedade, 24(1), 23-33. https://doi.org/10.1590/S0104-12902015000100002

Modesto, P. (2014). Notas para um debate sobre o princípio da eficiência. Revista do Serviço Público, 51(2), 105-119. https://doi.org/10.21874/rsp.v51i2.328

Moritz, S., \& Bartz-Beielstein, T. (2017). ImputeTS: Time Series Missing Value Imputation in R. The R Journal, 9(1), 207-218. 10.32614/RJ-2017-009.

OMS, O. M. da S. (2010). Relatório mundial da saúde: financiamento dos sistemas de saúde: o caminho para a cobertura universal. Retrieved from www. who.int/whr/2010/whr10_pt.pdf

OPAS/OMS, P.A. da S. O. (2012). Estratégia de Cooperação Técnica da OPAS/OMS com a República Federativa do Brasil, 2008-2012. Brasília: OPAS/OMS.

Purohit, B. C. (2014). Efficiency of social sector expenditure in India: a case of health and education in selected Indian states. Healthcare in Low-Resource Settings, 2(1). https://doi.org/10.4081/hls.2014.1866

Rezende, A. J., Slomski, V., \& Corrar, L. J. (2005). A gestão pública municipal e a eficiência dos gastos públicos: Uma investigação empírica entre as políticas públicas e o Índice de Desenvolvimento Humanao (IDH) dos municípios do estado de São Paulo. Revista Universo Contábil, 1(1), 24-40.

Santos, F. R., Jankoski, A. R. M. C., Oliveira, A. G., \& Rasoto, V. I. (2017). O Orçamento-Programa e a Execução das Políticas Públicas. Revista do Serviço Público. 68(1), 191-212.

Silva, M. C. da, Maciel, J. H. B. A., Chacon, M. J. M., \& Oliveira, A. (2008). Gastos com Saúde: uma Análise do Cumprimento da Emenda Constitucional no $29 / 2000$ pelos 100 Municípios Brasileiros mais Populosos, no período de 2000 a 2008. REUNIR - Revista de Administração, Contabilidade e Sustentabilidade, $2(1), 1-20$.

Sousa, M. da C. S. de., \& Ramos, F. S. (1999). Eficiência técnica e retornos de escala na produção de serviços públicos municipais: o caso do nordeste e do sudeste brasileiros. Revista Brasileira de Economia, 53(4), 433-461.

Souza, F. J. V. de., \& Barros, C. da C. (2013). Eficiência Na Alocação De Recursos Públicos Destinados a Assistência Hospitalar Nos Estados Brasileiros. Revista de Gestão, Finanças e Contabilidade, 3(1), 71-89.

Varela, P. S., Martins, G. D. A., \& Favero, L. P. L. (2010). Production efficiency and financing of public health: an analysis of small municipalities in the state of Sao Paulo-Brazil. Health Care Management Science, 13(2),112-123.https://doi.org/10.1007/s10729-009-9114-y

Wallmeler, F., Helmig, B., \& Feeney, M. K. (2018). Knowledge Construction in Public Administration: A Discourse Analysis of Public Value. Public Administration Review, 8 Nov, 488-499. https://doi.org/10.1111/puar.13005

Wilbert, M. D., \& D’Abreu, E. C. C. F. (2013). Eficiência dos Gastos Públicos na Educação: Análise dos Municípios do Estado de Alagoas. Advances in Scientific and Applied Accounting, 6(3), 348-372. 